\title{
Jahrestagung 2008
}

Die 68. Jahrestagung der Vereinigung der Deutschen Staatsrechtslehrer e.V. fand vom 1. bis 4. Oktober 2008 an der Friedrich-AlexanderUniversität Erlangen-Nürnberg statt, zum zweiten Mal in der Tagungsgeschichte der Vereinigung (nach 1959). Über 300 Mitglieder und 130 Gäste und Begleitpersonen konnten sich in großer Dankbarkeit über eine federführend von Max-Emanuel Geis als kooptiertem Vorstandsmitglied und seinem Team trefflich organisierte Tagung herzlich freuen, die auch in den Angeboten des nichtwissenschaftlichen Begleitprogramms die historische, kulturelle und wirtschaftliche Einbettung der Stadt Erlangen und ihrer Umgebung eindrucksvoll verdeutlichte.

Die wissenschaftlichen Begleitveranstaltungen am Mittwoch, dem 1. Oktober 2008 als Eröffnungstag waren zunächst durch Beratungen des Gesprächskreises „Europäisches Verfassungsrecht“ zur Sicherheit in der Europäischen Union mit Referaten von Markus Kotzur, Markus Möstl und Christoph Grabenwarter und des Gesprächskreises „Verwaltung“ zur Zukunft des Beamtentums mit Referaten von Gabriele KucskoStadlmayer und Hans Peter Bull bestimmt. Zu Beginn der anschließenden Mitgliederversammlung gedachten die Teilnehmer der im vorausgegangenen Jahr verstorbenen Mitglieder Manfred Abelein, Erich Küchenhoff und Klaus Vogel, denen die Vereinigung stets ein ehrendes Andenken bewahren wird. Die Vereinigung konnte im selben Zeitraum 15 neue Mitglieder begrüßen und ist ungeachtet einer abgeschwächten Wachstumsdynamik mit derzeit 670 Mitgliedern erneut so groß wie nie zuvor.

Das wissenschaftliche Programm der Jahrestagung widmete sich dem Generalthema "Erosion von Verfassungsvoraussetzungen“ anhand vier verschiedener Themenfelder, deren Probleme zentrale Funktionsvoraussetzungen des demokratischen Verfassungsstaates tangieren könnten. Die im Begriff „Erosion“ angelegte Spannung zwischen empirischer Beschreibung von dauerhaften, allmählichen und nur über längere Zeit merklichen Veränderungen und akzentuierter, kulturkritisch wertender Verfallsdiagnose öffnete den acht Berichterstattern ein breites Spektrum von Problemzugängen: Deren Kontraste vermittelten den Teilnehmern der Tagung reiche Anregungen und führten zu lebhaften Plenumsdiskussionen (unter Moderation von Christoph Engel bzw. Michael Holoubek), wie ihre nachstehende Dokumentation verdeutlicht.

Die Tagungsberatungen wurden eröffnet durch die Begrüßung des Sprechers des Fachbereichs Rechtswissenschaft der Rechts- und Wirtschaftswissenschaftlichen Fakultät, Herrn Kollegen Prof. Dr. Heinrich De Wall, und abgerundet durch abendliche Empfänge mit ihren Mög- 
lichkeiten zur vielfältigen Gesprächsfortsetzung: am Mittwoch im Erlanger Redoutensaal durch den Oberbürgermeister der Stadt Erlangen, Herrn Dr. Siegfried Balleis, und den Rektor der Friedrich-AlexanderUniversität Erlangen-Nürnberg, Herrn Prof. Dr. Karlheinz Grüske, am Donnerstag auf der Nürnberger Kaiserburg durch die Bayerische Staatsregierung, vertreten durch Frau Staatsministerin Dr. Renate Merk. Ein geselliger Freitagabend in der Palmeria (u. a. mit launigen Selbstreflexionen über die Vereinigung und ihre Mitglieder von Gunnar Folke Schuppert) und ein Ausflug am Samstag in die herbstliche Landschaft der Fränkischen Schweiz (u. a. mit einem Orgelkonzert von Max-Emanuel Geis in der Basilika Gößweinstein) beschlossen eine Jahrestagung, die durch eine von vielen Teilnehmerinnen und Teilnehmern als offen, diskursfreundlich und unverkrampft empfundene Atmosphäre die Freude aller Beteiligten an Staatsrechtslehre als Wissenschaft zum Ausdruck bringen konnte. 